\title{
Effects of Self-Esteem on the Associations of Infertility- Related Stress With Psychological Distress Among Chinese Women With Infertility: A Cross-Sectional Study
}

\author{
Chunying Cui \\ China Medical University

\section{Yu Wang} \\ China Medical University

\section{Lie Wang} \\ China Medical University \\ Xiaoxi Wang ( $\nabla$ xiaoxiwang@cmu.edu.cn ) \\ China Medical University
}

\section{Research}

Keywords: Infertility-related stress, Depression, Anxiety, Self-esteem, Infertility

Posted Date: May 24th, 2021

DOI: https://doi.org/10.21203/rs.3.rs-508041/v1

License: (c) (i) This work is licensed under a Creative Commons Attribution 4.0 International License. Read Full License 


\section{Abstract}

Background: Anxiety and depression are the most common occurring psychological distress in infertile patients. Our study aimed to evaluate the prevalence of depression and anxiety, and investigate the association of infertilityrelated stress with them among Chinese infertility patients. Meanwhile, the role of self-esteem in this association was explored as a positive psychological resource.

Methods: The investigation was conducted between December 2017 to May 2018. Questionnaires that measured infertility-related stress, self-esteem, depression, and anxiety were distributed to 536 female infertility patients in Shenyang, China. Hierarchical linear regression analyses were used to examine the mediating and moderating role of self-esteem. Baron and Kenny's technique, asymptotic, and resampling strategies were used to confirm the mediating role of self-esteem on the associations of infertility-related stress with depression and anxiety.

Results: The prevalence of depression and anxiety was $27.9 \%$ and $42.2 \%$ among Chinese female infertility patients. Infertility-related stress was positively associated with depression and anxiety, whereas self-esteem was negatively associated with depression and anxiety. Besides, the effect of infertility-related stress on depression and anxiety gradually decreases with the increase of self-esteem. Meanwhile, self-esteem partially mediated the association of infertility-related stress with depression and anxiety.

Conclusions: There was a high prevalence of psychological distress. Infertility-related stress and self-esteem were associated with psychological distress, which helped to explain why infertile women suffered higher prevalence of depression and anxiety. Besides, self-esteem mediated and moderated the effects of infertility-related stress on depression and anxiety. Interventions that focus on self-esteem may be effective and available resource to combat mental problems.

\section{Plain English Summary}

Infertility is regard as a negative life event, and therefore may result in adversely psychological consequences. Besides, it is widely known that women are more vulnerable to mental health than men due to infertility, particularly in developing countries (e.g., China). In the present study, female with infertility were investigated for the prevalence of depression and anxiety, and its association with demographic and clinical factors, stress-related infertility and self-esteem.

All participants were from Shenyang Women's and Children's Hospital, China from December 2017 to May 2018. Consented patients would be asked to completed questionnaire including demographic and clinical information, stress-related infertility and self-esteem.

Among 548 eligible participants, 536 effective responses were received (97.8\%) in present study. The prevalence of depression was 27.9\% (possible depression:20.3\%; probable depression:7.6\%) and anxiety was $42.2 \%$ (possible depression:23.5\%; probable depression:18.7\%) in female infertility. In addition, infertile women with sleep disorders and more stress related to infertility were more vulnerable to experience depression and anxiety while they with high level of self-esteem could buffer and decrease stress-related infertility and thereby effectively cope with psychological distress.

In conclusion, infertile women were at high risk for psychological distress. Therefore, psychological counseling should be integrated into in vitro fertilization treatment or other treatment methods to help infertile women cope 
with mental problems. In addition, Psychological intervention based on self-esteem should be introduced by clinician and nurses for women with infertility.

\section{Background}

Infertility is a disease and social problem that the World Health Organization (WHO) defines as "a disease of the reproductive system defined by the failure to achieve a clinical pregnancy after 12 months or more of regular unprotected sexual intercourse" [1], which threatens to approximately 45 million couples worldwide and the number is increasing annually [2]. Previous studies reported that $15.5 \%$ of reproductive-age women in the United States suffered from infertility [3], 24\% in France [4]. In China, relevant large-scale epidemiological studies revealed that the prevalence of infertility varies widely, ranging from 6.7-25\% among women in reproductive age [5-7].

In addition, prior studies have shown that women with infertility seem to be vulnerable to suffer from mental disorders caused by stress related to infertility, compared with male $[8,9]$. The impact of infertility on women's mental health is profound throughout their life, including emotional, spiritual, sexual, and physical aspects. Moreover, infertility diseases can result in low self-esteem, guilt and loneliness, social isolation, even psychological stress [10]. Depression and anxiety are the most common mental disorders in infertility patients [11]. Studies found that $14.7 \%$ infertile women suffered from anxiety in Italian population while men were $4.5 \%$ [12]. Besides, some scholars found an increased symptom load where $10-50 \%$ of subjects reported mild to moderate depression [13], major depression in 17\% of women [14]. Psychological disorders in women with infertility are associated with high rates of problematic personality traits like neuroticism [15], and low quality of life [16]. What's worse is that both depression and anxiety could result in decreasing fertility and rate of pregnancy in patients with assisted reproductive technology [17]. Therefore, early identing the influencing factors, screening high risk population and managing strategies may effectively decrease the negative consequences of the mood disorders in female infertility.

Research found that infertility-related stress has been identified significantly increasing both anxiety and depression in women with infertility $[18,19]$. Infertility-related stress refers to perceived stresses from one's social networks, marital relationship, sexual domain, rejection of childfree lifestyle as well as need for parenthood due to the infertility [20]. Actually, female infertility who are faced with infertile diagnosis may trigger some negative thoughts concerning the importance of rejection of childfree lifestyle and need for parenthood all their life. In addition, it may involve in feelings of stigma and social isolation from family and friends, reducing sexual pleasure and difficulty in discussing infertility with couple, which induces higher level of infertility-related stress. Furthermore, infertile treatment leads to heavy psychological and physical stress. Therefore, women with high level of infertility-related stress can be inclined to suffer from mood disorders.

Although the association of both depression and anxiety with infertility-related stress have been examined among female infertility patients $[18,19]$, the underlying connection still remain unclear. Over years, positive psychology is being increasingly used to prevent and treat mental health problems. Self-esteem is a term that reflects a person's overall evaluation or appraisal of her or his own worth, which encompasses beliefs and emotions such as triumph, despair, pride or shame [21]. Low level of self-esteem results in a series of psychological or physical disorders. Selfesteem has been found to be negatively related to depression among infertile couples [22, 23]. Besides, other study found that self-esteem could mediate the relationship between mindfulness and depression in general population [24]. Another study self-esteem moderated the negative impact of body-related shame and guilt on mental disorders among young adults [25]. Since self-esteem as a protective factor can effectively buffer against the adverse impact 
of negative power on mental health. In other words, self-esteem may affect the negative effect of infertility-related stress on depression or anxiety. Therefore, we thought self-esteem may affect the association of infertility-related stress with depression and anxiety in women with infertility.

Nevertheless, the role of self-esteem in the relationships of infertility-related stress with depression and anxiety has still not yet been explored among female infertility patients. Therefore, our study aimed to tested the association of infertility-related stress with psychological disorders and examined the role of self-esteem in the association between infertility-related stress and depression in women with infertility.

\section{Methods}

\section{Study Design and Data Collection}

Our study used a cross-sectional design, and was conducted in Liaoning Province, China from December 2017 to May 2018. All participants were from Shenyang Women's and Children's Hospital. Participants included Women who were diagnosed with infertility, could fluently communicate in Chinese, and aged over 18. Participants excluded women who had other major diseases at the present stage, psychiatric history in the past, and cognitive impairments. Consented patients would be asked to completed questionnaire. Among 548 eligible participants, 536 effective responses were received (97.8\%) in present study. Reasons for five non-participants were health problems, and seven questionnaires with missing data were excluded.

\section{Measurement of Depression and Anxiety}

The Hospital Anxiety and Depression Scale (HADS) was one of the most commonly used instruments worldwide for assessing anxiety and depression in clinical patients with physical problems [26]. The HADS is a 14-items scale and consists of two subscales including anxiety and depression, with seven items each. Each of the items is scored on a Likert scale ( 0 as not at all, 3 as very much indeed). The global score in each subscale was 0 to 21 . Higher score was related to higher anxiety and depression. HADS-A or HADS-D score $\geq 8$ indicates possible depression and anxiety, and score $\geq 10$ means probable depression and anxiety [26]. The current version had adequate reliability and validity among women with infertility [27]. The Cronbach's alpha coefficient for HADS was 0.840 (HADS-D: 0.693, HADS-A: 0.779).

\section{Measurement of Infertility-related Stress}

Newton et al compiled Fertility Problem Inventory (FPI), which aims to measure the degree of stress associated with infertility. FPI contains five dimensions (rejection of a childfree lifestyle, social concern, sexual concern, relationship concern, the need for parenthood), and 46 items [20]. Peng et al (2011) translated FPI from English to Chinese, and reported good validity and reliability for FPI in Chinese population [28]. Each of the items is scored on a Likert scale ( 1 as not agree, 6 as totally agree). The global FPI score was 46 to 276 . Higher score was related to the higher degree of stress associated with infertility. The Chinese version of FPI reported a Cronbach's alpha of 0.887 in the current study.

\section{Measurement of Self-esteem}

Rosenberg (1965) developed Rosenberg self-esteem scale (RSES), which was used to test self-esteem [29]. The Chinese version was translated by Cheng et al [30] and consists of 10 items. Participants rated each item on a 4point Likert scale (from "strongly agree" to "strongly disagree"). The current version was used in previous studies $[30,31]$. The Chinese version of FPI reported a Cronbach's alpha of 0.749 in the current study. 


\section{Demographic and Clinical Information}

We collected the demographic variables of patients including age, residence, educational level, income, times of exercise weekly, sources of stress and life event. Residence was divided into rural area and urban area. Educational level was divided into senior high school or lower, junior college and bachelor degree or higher. Income was divided into $<4,000$ and $\geq 4,000$ yuan. Times of exercise weekly was categorized into $0,1-2$ and $3-4$. Sources of stress was divided into the family, oneself and others (friend or colleague). Life event was divided into yes or no. We also surveyed the clinical variables including causes of infertility and past histories (pregnancy, infection, operation and infertility treatment). Causes of infertility were categorized as male factors (oligospermia, erectile dysfunction, etc.), female factors (e.g., endometriosis, ovulation disorders), and unexplained reason. Past histories were categorized into yes or no.

\section{Statistical Analyses}

In this study, SPSS 22.0 was used to analyzed data. One-way ANOVA or independent-group t-tests was used to examine the difference of depression and anxiety in categorical variables. Correlations was examined by using Pearson's correlation among continuous variables. Hierarchical regression analysis was used to investigated the effects of independent variables on depression and anxiety and explored the effect of self-esteem in this relationship. In model 1, all demographic and clinical variables which were significantly related to depression or anxiety in univariate analyses were entered; in model 2, the infertility-related stress was entered; in model 3, selfesteem variable was added; in model 4 , the production of infertility-related stress and self-esteem was added. There was the moderating role of self-esteem if the interaction was significant in regression model [32]. Asymptotic and resampling strategies, developed by Preacher and Hayes [33], were used to examine the mediating roles of positive psychological resources (a*b product) on the association between infertility-related stress and psychological outcomes including depression and anxiety. The bootstrap estimate was based on 5000 bootstrap samples. The bias-corrected and accelerated 95\% confidence interval (BCa 95\% Cl) for each a*b product was calculated, and a $\mathrm{BCa} 95 \% \mathrm{Cl}$ excluding 0 indicated a significant mediating role.

All statistical tests were two-sides $(a=0.05)$.

\section{Results}

\section{Descriptive statistics}

Table 1 showed demographic and infertility characteristics of subjects and distributions of depression and anxiety. The mean age of the subjects was 32.77 years ( $S D=4.42$, ranged from 22 to 47). Education level, income, sleep disorders, times of exercise weekly and history of infertility treatment were related to depression $(p<0.05)$. With regard to the level of education and income, patients with higher educational level and income had lower score of depression. There were 315 (58.8\%) patients who received infertile treatment and the scores of depressions were higher than patients without treatment. Approximately half of women (48.7\%) suffered from sleep disorders and the level of depression were higher than women without sleep disorders. Patients (46.5\%) without exercise habit suffered from higher level of depression than women with exercise. Sleep disorders and history of infertility treatment were associated with anxiety $(p<0.05)$. 
Table 1

Distribution of characteristics and univariate analyses of depression and anxiety

\begin{tabular}{|c|c|c|c|c|c|}
\hline \multirow[t]{2}{*}{ Variables } & \multirow[t]{2}{*}{$\mathbf{N}(\%)$} & Depression & \multirow[t]{2}{*}{$P$} & Anxiety & \multirow[t]{2}{*}{$P$} \\
\hline & & Mean \pm SD & & Mean \pm SD & \\
\hline Age & & & 0.931 & & 0.177 \\
\hline $20-29$ & 128(23.9) & $5.41 \pm 3.41$ & & $7.42 \pm 3.96$ & \\
\hline $30-39$ & $365(68.1)$ & $5.35 \pm 3.43$ & & $7.00 \pm 3.75$ & \\
\hline$>40$ & $43(8.0)$ & $5.19 \pm 3.30$ & & $6.21 \pm 3.76$ & \\
\hline Residence & & & 0.419 & & 0.536 \\
\hline Rural area & $111(20.7)$ & $5.59 \pm 3.21$ & & $7.23 \pm 3.59$ & \\
\hline Urban area & $425(79.3)$ & $5.29 \pm 3.46$ & & $6.99 \pm 3.80$ & \\
\hline Education level & & & 0.008 & & 0.395 \\
\hline Senior high school or lower & $224(41.8)$ & $5.86 \pm 3.38$ & & $7.18 \pm 3.72$ & \\
\hline Junior college & $114(21.3)$ & $5.25 \pm 3.35$ & & $6.61 \pm 3.86$ & \\
\hline Bachelor degree or above & 198(36.9) & $4.83 \pm 3.41$ & & $7.12 \pm 3.74$ & \\
\hline Income (RMB, yuan) & & & 0.001 & & 0.413 \\
\hline$<4000$ & $144(26.9)$ & $6.19 \pm 3.29$ & & $7.26 \pm 3.62$ & \\
\hline$\geq 4000$ & 392(73.1) & $5.04 \pm 3.40$ & & $6.96 \pm 3.81$ & \\
\hline History of pregnancy & & & 0.616 & & 0.588 \\
\hline No & $283(52.8)$ & $5.28 \pm 3.48$ & & $6.95 \pm 3.81$ & \\
\hline Yes & $253(47.2)$ & $5.43 \pm 3.33$ & & $7.13 \pm 3.70$ & \\
\hline Cause of infertility & & & 0.116 & & 0.227 \\
\hline Female factor & $331(61.8)$ & $5.51 \pm 3.20$ & & $7.26 \pm 3.71$ & \\
\hline Male factor & $93(17.4)$ & $4.69 \pm 3.86$ & & $6.65 \pm 4.10$ & \\
\hline Unexplained & $112(20.9)$ & $5.45 \pm 3.58$ & & $6.71 \pm 3.60$ & \\
\hline History of infection & & & 0.535 & & 0.878 \\
\hline No & $496(92.5)$ & $5.33 \pm 3.44$ & & $7.03 \pm 3.75$ & \\
\hline Yes & $40(7.5)$ & $5.68 \pm 3.03$ & & $7.13 \pm 3.96$ & \\
\hline History of operation & & & 0.126 & & 0.141 \\
\hline No & $316(59.0)$ & $5.17 \pm 3.44$ & & $6.85 \pm 3.75$ & \\
\hline Yes & $220(41.0)$ & $5.60 \pm 3.36$ & & $7.30 \pm 3.77$ & \\
\hline History of infertility treatment & & & 0.019 & & 0.007 \\
\hline No & $221(41.2)$ & $4.94 \pm 3.28$ & & $6.52 \pm 3.52$ & \\
\hline
\end{tabular}




\begin{tabular}{|c|c|c|c|c|c|}
\hline \multirow[t]{2}{*}{ Variables } & \multirow[t]{2}{*}{$\mathbf{N}(\%)$} & Depression & \multirow[t]{2}{*}{$P$} & Anxiety & \multirow[t]{2}{*}{$P$} \\
\hline & & Mean \pm SD & & Mean \pm SD & \\
\hline Yes & $315(58.8)$ & $5.64 \pm 3.47$ & & $7.40 \pm 3.88$ & \\
\hline Life event & & & 0.403 & & 0.087 \\
\hline No & 404 & $5.28 \pm 3.39$ & & $6.88 \pm 3.65$ & \\
\hline Yes & 132 & $5.57 \pm 3.47$ & & $7.52 \pm 4.05$ & \\
\hline Sleep disorders & & & $<0.001$ & & $<0.001$ \\
\hline No & $275(51.3)$ & $4.50 \pm 3.23$ & & $5.86 \pm 3.46$ & \\
\hline Yes & $261(48.7)$ & $6.25 \pm 3.37$ & & $8.28 \pm 3.67$ & \\
\hline Times of exercise weekly & & & 0.006 & & 0.083 \\
\hline 0 & $249(46.5)$ & $5.86 \pm 3.44$ & & $7.42 \pm 3.82$ & \\
\hline $1-2$ & 188(35.1) & $4.95 \pm 3.41$ & & $6.76 \pm 3.66$ & \\
\hline $3-4$ & $99(18.5)$ & $4.85 \pm 3.18$ & & $6.61 \pm 3.72$ & \\
\hline Sources of stress & & & 0.764 & & 0.095 \\
\hline The family & $166(31.0)$ & $5.25 \pm 3.48$ & & $7.17 \pm 3.66$ & \\
\hline Oneself & $293(54.7)$ & $5.34 \pm 3.37$ & & $6.77 \pm 3.81$ & \\
\hline Others (friend or colleague) & $77(14.4)$ & $5.60 \pm 3.43$ & & $7.78 \pm 69$ & \\
\hline
\end{tabular}

Table 2 presented the levels of depression, anxiety, infertility-related stress, self-esteem and age. The mean score of depression and anxiety were 5.35 (SD = 4.42) and $7.04(\mathrm{SD}=3.76)$, respectively. In addition, the prevalence of depression was $27.9 \%$ (possible depression:20.3\%; probable depression:7.6\%) and anxiety was $42.2 \%$ (possible depression:23.5\%; probable depression:18.7\%) in female infertility.

Table 2

Description statistics for continuous variables

\begin{tabular}{|lllll|}
\hline Variables & Mean & SD & Range & $\mathbf{n}(\%)$ \\
\hline Age & 32.77 & 4.42 & $22-47$ & \\
\hline Depression & 5.35 & 3.41 & $0-16$ & \\
\hline $8 \leq$ Scores $\leq 10$ & & & & $109(20.3)$ \\
\hline $11 \leq$ Scores $\leq 21$ & & & & $41(7.6)$ \\
\hline Anxiety & 7.04 & 3.76 & $0-18$ & \\
\hline $8 \leq$ Scores $\leq 10$ & & & & $126(23.5)$ \\
\hline $11 \leq$ Scores $\leq 21$ & & & & $100(18.7)$ \\
\hline Infertility-related stress & 140.41 & 30.29 & $61-226$ & \\
\hline Self-esteem & 29.08 & 3.94 & $17-40$ & \\
\hline
\end{tabular}




\section{Correlations among continuous variables}

Table 3 indicated the correlations between infertility-related stress, self-esteem and depression. Infertility-related stress was significantly and positively associated with depression and anxiety $(p<0.01)$, whereas self-esteem was significantly and negatively associated with depression and anxiety $(p<0.01)$. Age was significantly and negatively associated with anxiety $(p<0.05)$.

Table 3

Correlations among continuous variables

\begin{tabular}{|llllll|}
\hline Variables & $\mathbf{1}$ & $\mathbf{2}$ & $\mathbf{3}$ & $\mathbf{4}$ & $\mathbf{5}$ \\
\hline 1.Age & 1 & & & & \\
\hline 2.Depression & -0.11 & 1 & & & \\
\hline 3.Anxiety & $-0.103^{*}$ & $0.661^{* \star}$ & 1 & & \\
\hline 4.Infertility-related stress & -0.059 & $0.456^{* \star}$ & $0.451^{\star \star}$ & 1 & \\
\hline 5. Self-esteem & $0.163^{\star \star}$ & $-0.444^{\star \star}$ & $-0.406^{\star \star}$ & $-0.445^{\star \star}$ & 1 \\
\hline
\end{tabular}

${ }^{*} p<0.05,{ }^{* *} p<0.01$

\section{Hierarchical regression analysis}

Tables 4 and 5 showed the predictors of depression and anxiety based on hierarchical regression analysis. Based on the results of univariate analyses, age, education, income, sleep disorders, time of exercise weekly and history of infertility treatment were defined as control variables in regression model 1 of depression, and age, sleep disorders and history of infertility treatment were entered in regression model 1 of anxiety. In model 2, infertility-related stress was significantly and positively associated with depression $(\beta=0.339, P<0.001)$ and anxiety $(\beta=0.401, P<0.001)$. Self-esteem (depression: $\beta=-0.280, P<0.001$; anxiety: $\beta=-0.225, P<0.001$ ) remained in model 3 and together with infertility-related stress, the model explained $31.4 \%$ of the variance in depression and anxiety was $31.0 \%$. In model 4 , interaction term was significantly and negatively associated with depression $\left(F=29.203, \beta=-0.099\right.$, adjusted $R^{2}=$ $0.322, P<0.001)$, which showed that the effect of infertility-related stress on depression decreased with the increase of self-esteem in infertile women. For anxiety, interaction term was significantly in model $4(F=43.105, \beta=-0.133$, adjusted $\left.R^{2}=0.321, P<0.001\right)$, which showed that the effects of infertility-related stress on anxiety gradually decreases with the increase of self-esteem in infertile women. 
Table 4

Hierarchical linear regression analyses of the factors associated with depression

\begin{tabular}{|c|c|c|c|c|}
\hline \multirow[t]{2}{*}{ Variables } & \multicolumn{4}{|l|}{ Depression } \\
\hline & Model $1(\beta)$ & Model 2( $\beta)$ & Model $3(\beta)$ & Model $4(\beta)$ \\
\hline Age & 0.023 & 0.036 & 0.068 & 0.064 \\
\hline Education level & -0.082 & -0.051 & -0.006 & -0.003 \\
\hline Income & $-0.134^{\star \star \star}$ & $-0.093^{*}$ & $-0.073^{*}$ & -0.068 \\
\hline History of infertility treatment & $0.088^{*}$ & 0.057 & 0.056 & 0.053 \\
\hline Sleep disorders & $0.245^{\star \star \star}$ & $0.188^{\star \star \star}$ & $0.167^{\star \star \star}$ & $0.167^{\star \star \star}$ \\
\hline Times of exercise weekly & $-0.106^{\star}$ & $-0.076^{*}$ & -0.068 & -0.069 \\
\hline Infertility-related stress & & $0.399^{\star \star \star}$ & $0.288^{\star \star \star}$ & $0.297^{\star \star \star}$ \\
\hline Self-esteem & & & $-0.280^{\star \star \star}$ & $-0.294^{\star \star \star}$ \\
\hline Interaction & & & & $-0.099^{\star *}$ \\
\hline$F$ & 11.574 & 27.275 & 31.547 & 29.203 \\
\hline Adjusted $R^{2}$ & 0.106 & 0.256 & 0.314 & 0.322 \\
\hline
\end{tabular}

${ }^{*} p<0.05,{ }^{* *} p<0.01,{ }^{\star \star *} p<0.001$

Table 5

Hierarchical linear regression analyses of the factors associated with anxiety

\begin{tabular}{|c|c|c|c|c|}
\hline \multirow[t]{2}{*}{ Variables } & \multicolumn{4}{|l|}{ Anxiety } \\
\hline & Model $1(\beta)$ & Model 2( $\beta)$ & Model $3(\beta)$ & Model $4(\beta)$ \\
\hline Age & $-0.084^{*}$ & -0.064 & -0.034 & -0.039 \\
\hline History of infertility treatment & $0.102^{*}$ & 0.069 & 0.069 & 0.066 \\
\hline Sleep disorders & $0.312^{\star \star \star}$ & $0.251^{\star \star \star}$ & $0.234^{\star \star \star}$ & $0.233^{\star \star \star}$ \\
\hline Infertility-related stress & & $0.401^{\star \star \star}$ & $0.306^{\star \star \star}$ & $0.315^{\star \star \star}$ \\
\hline Self-esteem & & & $-0.225^{\star \star \star}$ & $-0.240^{\star \star \star}$ \\
\hline Interaction & & & & $-0.133^{\star *}$ \\
\hline$F$ & 24.411 & 50.783 & 49.011 & 43.105 \\
\hline Adjusted $R^{2}$ & 0.116 & 0.271 & 0.310 & 0.321 \\
\hline
\end{tabular}

${ }^{*} p<0.05,{ }^{* *} p<0.01,{ }^{* \star} p<0.001$ 
According to Table 4 and 5, infertility-related stress was positively and significantly related to depression $(\beta=0.339$, $P<0.001)$ and anxiety $(\beta=0.401, P<0.001)$ in model 2 ; in model 3 , self-esteem was negatively correlated with depression $(\beta=-0.280, P<0.001)$ and anxiety $(\beta=-0.225, P<0.001)$ and the effect of infertility-related stress on depression $(\beta=0.288, P<0.001)$ and anxiety $(\beta=0.306, P<0.001)$ was smaller compared with that in model 2 , which indicated that infertility-related stress probably could become a partial mediator on the association of WPV with depression and anxiety.

Given that, for Table 6, self-esteem mediated the association of infertility-related stress with depression $(a * b=$ 0.1109 , BCa 95\% Cl: 0.0770,0.1495) and anxiety ( $\mathrm{a} * \mathrm{~b}=0.0956, \mathrm{BCa} 95 \% \mathrm{Cl}: 0.0622,0.1327)$. Exactly, self-esteem played a partial mediating effect in the relationship of infertility-related stress with depression $\left(c^{\prime}=0.1133, P<0.001\right)$ and anxiety $\left(c^{\prime}=0.1133, P<0.001\right)$.

Table 6 Test of mediation model

\begin{tabular}{|c|c|c|c|c|c|c|c|}
\hline$x$ & M & $\mathrm{Y}$ & c & a & b & $c^{\prime}$ & a*b (BCa 95\%) \\
\hline \multirow{2}{*}{$\begin{array}{l}\text { Infertility- } \\
\text { related } \\
\text { stress }\end{array}$} & \multirow{2}{*}{$\begin{array}{l}\text { Self- } \\
\text { esteem }\end{array}$} & Depression & $0.3990^{\star \star \star}$ & $-0.3962^{\star \star \star}$ & $-0.2799^{\star \star \star}$ & $0.2881^{\star \star \star}$ & $0.1109(0.0770,0.1495)$ \\
\hline & & Anxiety & $0.4014^{\star \star \star}$ & $-0.4242^{\star \star \star}$ & $-0.2253^{\star \star \star}$ & $0.3058^{\star \star \star}$ & $0.0956(0.0622,0.1327)$ \\
\hline
\end{tabular}

${ }^{\star} p<0.05,{ }^{* \star} p<0.01,{ }^{\star \star *} p<0.001$

a: associations of infertility-related stress with self-esteem

b: associations of self-esteem with depression or anxiety

c: direct associations of infertility-related stress with depression or anxiety

c': indirect associations of infertility-related stress with depression or anxiety

Age, education level, income, history of infertility treatment, sleep disorders and times of exercise weekly are covariates for depression.

Age, history of infertility treatment, sleep disorders are covariates for anxiety.

\section{Discussion}

In this study, the prevalence of depression and anxiety was 27.9\% (possible depression: 20.3\%; probable depression: 7.6\%) and $42.2 \%$ (possible depression: $23.5 \%$; probable depression: $18.7 \%$ ) respectively, which revealed significantly worse psychological status in infertile women in terms of depression and anxiety. In addition, the prevalence of comorbidity was $22.4 \%$ in this study, indicating that depression and anxiety coexisted in Chinese women with infertility. This result was similar to previous studies [22,34]. This situation should be remarkable because comorbidity of depression and anxiety showed severer symptoms, lower compliance for treatment and poorer prognosis than patients with a single mental problem in infertile women.

Besides, compared with other studies, the prevalence of mood disorders in our study was higher. For instance, Chiaffarino et al reported that the prevalence of depression and anxiety was $17.9 \%$ and $14.7 \%$ among women with undergoing assisted reproductive treatment in an Italian infertility department based on 1000 couples [12]. Biringer 
et al found that $17.0 \%$ of infertile women had anxiety and depression was $7.0 \%$ in study of Norway based on 12584 Norwegian women from 1995 to 1997 [35]. In addition, Peterson et al showed that depression was reported in 11.6\% of 1131 infertile women in Denmark [36]. Several reasons might explain this difference of prevalence in depression and anxiety among women with infertility. At first, the results of our study might be overrated because of small sample size and high data fluctuation. Furthermore, above research that mainly were conducted in high-income or developed countries had lower level of depression and anxiety compared with developing or low-countries, like China. People in developed or higher income countries tend to possess more adequate health resources and health literacy to deal with mental problems. At last, the continuity of ethnicity is one of the crucial cores in terms of Chinese traditional culture, and Chinese culture places great emphasis on female reputation. However, infertile women fail to achieve a clinical pregnancy. Thus, it is very imperative to find out the crucial influencing factors and targeted solutions to improve mental health in Chinese women with infertility.

According to the results of univariate analyses, our study found income, education, history of infertility treatment, sleep disorders and exercise were associated with depression and anxiety. Education has been significantly associated with the development of depression, which was in line with the findings of previous studies [35, 37]. High education level was related to lower depression because women with higher education may have different life roles or social/work opportunities than becoming mothers and fixing themselves on this life goal. This aspect may allow them to accept infertility in a way to some extent less distressing. Previous study has also found that monthly family income was important factor of depression [38]. Family income has been put an additional strain on psychological and psychological status of couples struggling with infertility [39]. The improvement in educational level and family income might increase the feelings of control and self-confidence in female infertility patients, which relieved depression to some extent. Several articles reported that (mostly unsuccessful) infertility treatments increased the probability of negative emotions [40], especially in depression and anxiety [38]. Patients with high income had low scores of depressions in our study. Besides, recent study poor sleep quality contributed to the development of depression and anxiety in infertile women with vitro fertilization treatment [41].

Results of this study showed that after adjusting for control variables, infertility-related stress was an important factor affecting mood disorders in infertile women. More specifically, infertility-related stress was significantly and positively related to depression and anxiety, which was in accordance with several studies. Infertility diagnosis as a neglect stressor increases the feelings of stigma and guilt, which induces series of negative emotions. According to the stress-health theory, high and long-time stress could damage an individual's immune system, and even lead to psychological and physical vulnerability [42]. Other studies concerning infertility in women reported that infertilityrelated stress exhibited significant main effect on psychological distress (e.g., depression and anxiety) [18, 19]. Besides, infertility-related stress could decrease the well-being and increase marital conflicts [43, 44]. Noticeably, the mean score of infertility-related stress in our study was higher than other countries $[45,46]$. The possible interpretation may be the differences of traditional culture. In China, couples are more inclined to give priority to the needs of family members (e.g., having babies) over their own interest, and they without children are regard as losers. Therefore, female infertility might become more vulnerable to comments about a child, and easily induce uncomfortable feelings, which results in more mental problems.

An increasing number of scholars have become interested in the effects of positive psychological resources on mental health outcomes. Our research extended this research direction to the integrative and independent contributions of these positive psychological to depression in heterogeneous sample of female infertility patients. Self-esteem accounted for a mediating proportion of variance in depression (27.8\%) and anxiety (23.8\%). As an important inner resource for psychological and social functioning, self-esteem was found to be negatively

Page $11 / 16$ 
correlated with trait of depression and anxiety, which were consistent with the findings in previous study [22, 23]. Moreover, self-esteem was independently associated with infertility-related stress, indicating that each form of positive expectations had a unique association with infertility-related stress. These results contributed to the understanding that self-esteem was positive resource for combating mental problems and encouraged us to explore the mediating roles of positive psychological resources in the relationship of infertility-related stress with depression and anxiety in this population.

In the present study, self-esteem played a partial mediating role in the relationship of infertility-related stress with depression and anxiety. In other words, women with fertility who perceived more infertile-related stress would be more likely to experience lower levels of positive psychological resources which in turn increased the possibilities of developing depression. Compared with decreasing patient's infertility-related stress, it is a more positive and strategic way for people to develop programs to increase the inner positive resources of their patients, thus to enhance the mental health in the long run. Besides, our study found self-esteem moderated the effect of infertilityrelated stress on depression and anxiety in female infertility. Simple slope analysis showed that when fertile women possessed high self-esteem, depression and anxiety significantly decreased with the high levels of infertility-related stress, compared with low level of infertility-related stress. In other words, women with fertility possess high level of self-esteem, they can better maintain mental health even at a higher level of infertility-related stress. Therefore, resilience should be emphasized and developed to deal with mental problems.

\section{Implications}

According to our findings, some valuable implications should be highlight to cope with psychological distress and improve mental health nursing in infertile women. First, it is urgent to evaluate patients' psychological status during the diagnosis and treatment of infertility. Second, psychological counseling should be integrated into in vitro fertilization treatment or other treatment methods to help infertile women cope with mental problems. Third, the family are one of the main sources of stress, thus, they, especially spouses should provide support and understanding in mental nursing, which can relief patients' negative mood. At last, psychological intervention based on self-esteem should be introduced by clinician and nurses for women with infertility. For instance, Ribeiro et al (2020) proposed "Strengthening Self-Esteem" intervention through the use of social software was effective for improving increase the level of self-esteem and self-efficacy [47]. In addition, such interventions help spread knowledge in mental health nursing and build mentally healthier individuals.

\section{Limitations}

There were several limitations in the current study. First, our study was from a cross-sectional design. Longitudinal studies are required to replicated our results in further research. Second, these associations in our study may be underestimated or overestimated due to study based on self-report measure tools. Third, the present study only recruited a non-random sample of infertile women in Liaoning province, which might result in self-selection bias in participants, and limit the generalizability of findings in our study.

\section{Conclusions}

To summarize, our findings revealed that infertility-related stress was positively associated with depression and anxiety among Chinese female infertility patients. In addition, self-esteem mediated and moderated the effects of infertility-related stress on depression and anxiety. Thus, interventions to decrease Chinese patients' infertility-

Page 12/16 
related stress and to enhance their positive psychological resources should be developed and put into practice in Chinese women with fertility.

\section{Declarations}

\section{Author contributions}

CYC and XXW conceived and designed the survey. YW and LW performed the investigation. CYC and XXW analyzed the data. CYC wrote the original paper. XXW revised the paper. All authors read and approved the final manuscript.

\section{Acknowledgment}

We would like to thank all the patients who voluntarily participated in this study and

research assistants who performed the data collection.

\section{Funding}

There was no funding support.

\section{Competing interests}

None

\section{Compliance with ethical standards}

\section{Data availability statement}

The dataset in this study is available from the corresponding author on reasonable request.

\section{Consent for publication}

Not applicable

\section{Ethical approval}

The study was approved by the Ethics Committee on Human Experimentation of China Medical University, and the study procedures were in accordance with ethical standards.

\section{References}

1. Zegers-Hochschild F, Adamson GD, de Mouzon J, Ishihara O, Mansour R, Nygren K, Sullivan E, Vanderpoel S, International Committee for Monitoring Assisted Reproductive Technology; World Health Organization. International Committee for Monitoring Assisted Reproductive Technology (ICMART) and the World Health Organization (WHO) revised glossary of ART terminology, 2009. Fertil Steril. 2009;92(5):1520-4. doi:10.1016/j.fertnstert.2009.09.009.

2. Mascarenhas MN, Flaxman SR, Boerma T, Vanderpoel S, Stevens GA. National, regional, and global trends in infertility prevalence since 1990: a systematic analysis of 277 health surveys. PLoS Med, 2012, 9(12): e1001356. doi: 10.1371/journal. pmed.1001356. 
3. Thoma ME, McLain AC, Louis JF, King RB, Trumble AC, Sundaram R, Buck Louis GM. Prevalence of infertility in the United States as estimated by the current duration approach and a traditional constructed approach. Fertil Steril. 2013;99(5):1324-31.e1. doi:10.1016/j.fertnstert.2012.11.037.

4. Slama R, Hansen OK, Ducot B, Bohet A, Sorensen D, Giorgis Allemand L, Eijkemans MJ, Rosetta L, Thalabard JC, Keiding N, Bouyer J. Estimation of the frequency of involuntary infertility on a nation-wide basis. Hum Reprod. 2012;27(5):1489-98. doi:10.1093/humrep/des070.

5. Zhou Z, Zheng D, Wu H, Li R, Xu S, Kang Y, Cao Y, Chen X, Zhu Y, Xu S, Chen ZJ, Mol BW, Qiao J. Epidemiology of infertility in China: a population-based study. BJOG. 2018;125(4):432-41. doi:10.1111/1471-0528.14966.

6. Liu J, Larsen U, Wyshak G. Prevalence of primary infertility in China: in-depth analysis of infertility differentials in three minority province/autonomous regions. J Biosoc Sci. 2005;37(1):55-74.

doi:10.1017/s0021932003006461.

7. Junqing W, Qiuying Y, Jianguo T, Wei Y, Liwei B, Yuxian L, Yumei Z, Kangshou Y, Weiqun L, Lu C, Ersheng G. Reference value of semen quality in Chinese young men. Contraception. 2002;65(5):365-8. doi:10.1016/s0010-7824(02)00281-0.

8. Reis S, Xavier MR, Coelho R, Montenegro N. Psychological impact of single and multiple courses of assisted reproductive treatments in couples: a comparative study. Eur J Obstet Gynecol Reprod Biol. 2013;171(1):61-6. doi:10.1016/j.ejogrb.2013. 07.034.

9. Schaller MA, Griesinger G, Banz-Jansen C. Women show a higher level of anxiety during IVF treatment than men and hold different concerns: a cohort study. Arch Gynecol Obstet. 2016;293(5):1137-45. doi:10.1007/s00404-016-4033-x.

10. Schmidt L. Social and psychological consequences of infertility and assisted reproduction - what are the research priorities? Hum Fertil (Camb). 2009;12(1):14-20. doi:10.1080/14647270802331487.

11. Covington SN, editor. Fertility Counseling: Clinical Guide and Case Studies. Cambridge: Cambridge University Press; 2015.

12. Chiaffarino F, Baldini MP, Scarduelli C, Bommarito F, Ambrosio S, D'Orsi C, Torretta R, Bonizzoni M, Ragni G. Prevalence and incidence of depressive and anxious symptoms in couples undergoing assisted reproductive treatment in an Italian infertility department. Eur J Obstet Gynecol Reprod Biol. 2011;158(2):235-41. doi:10.1016/j.ejogrb.2011.04.032.

13. Williams KE, Marsh WK, Rasgon NL. Mood disorders and fertility in women: a critical review of the literature and implications for future research. Hum Reprod Update. 2007;13(6):607-16. doi:10.1093/humupd/dmm019.

14. Chen TH, Chang SP, Tsai CF, Juang KD. Prevalence of depressive and anxiety disorders in an assisted reproductive technique clinic. Hum Reprod. 2004;19(10):2313-8. doi:10.1093/humrep/deh414.

15. Volgsten H, Ekselius L, Poromaa IS, Svanberg AS. Personality traits associated with depressive and anxiety disorders in infertile women and men undergoing in vitro fertilization treatment. Acta Obstet Gynecol Scand. 2010;89(1):27-34. doi: 00016340903447396.

16. Wilson JF, Kopitzke EJ. Stress and infertility. Curr Women's'. Hlth Rep. 2002;2:194-9.

17. Milazzo A, Mnatzaganian G, Elshaug AG, Hemphill SA, Hiller JE, Astute Health Study Group. Depression and Anxiety Outcomes Associated with Failed Assisted Reproductive Technologies: A Systematic Review and MetaAnalysis. PLoS One. 2016;11(11):e0165805. doi:10.1371/journal.pone.0165805.

18. Chaves C, Canavarro MC, Moura-Ramos M. The Role of Dyadic Coping on the Marital and Emotional Adjustment of Couples with Infertility. Fam Process. 2019;58(2):509-23. doi:10.1111/famp.12364. 
19. Zurlo MC, Cattaneo Della Volta MF, Vallone F. Factor structure and psychometric properties of the Fertility Problem Inventory-Short Form. Health Psychol Open. 2017;20(4(2):2055102917738657. doi:10.1177/2055102917738657.

20. Newton CR, Sherrard W, Glavac I. The Fertility Problem Inventory: measuring perceived infertility-related stress. Fertil Steril, 1999, 72(1):54-62. doi: 10.1016/ s0015-0282(99)00164-8.

21. Sharma S, Agarwala S. Self-Esteem and Collective Self-Esteem as Predictors of Depression. Journal of Behavioral Sciences. 2014;24:1.

22. El Kissi Y, Romdhane AB, Hidar S, Bannour S, Ayoubi Idrissi K, Khairi H, Ben Hadj Ali B. General psychopathology, anxiety, depression and self-esteem in couples undergoing infertility treatment: a comparative study between men and women. Eur J Obstet Gynecol Reprod Biol,2013,167(2):185-9. doi: j. ejogrb.2012.12.014.

23. Zayed AA, El-Hadidy MA. Sexual satisfaction and self-esteem in women with primary infertility. Middle East Fertility Society Journal,2020,25(1).

24. Bajaj B, Robins RW, Pande N. Mediating role of self-esteem on the relationship between mindfulness, anxiety, and depression. Personality Individual Differences. 2016;96:127-31. doi:10.1016/j.paid.2016.02.085.

25. Brunet J, Pila E, Solomon-Krakus S, Sabiston CM, O'Loughlin J. Self-esteem moderates the associations between body-related self-conscious emotions and depressive symptoms. J Health Psychol. 2019;24(6):83343. doi: 1359105316683786.

26. Zigmond AS, Snaith RP. The hospital anxiety and depression scale. Acta Psychiatr Scand. 1983;67(6):361-70. doi:10.1111/acp.1983.67.issue-6.

27. Bjelland I, Dahl AA, Haug TT, Neckelmann D. The validity of the Hospital Anxiety and Depression Scale. An updated literature reviews. J Psychosom Res. 2002;52(2):69-77. doi:10.1016/s0022-3999(01)00296-3.

28. Peng T, Coates R, Merriman G, Zhao Y, Maycock B. Testing the psychometric properties of Mandarin version of the fertility problem inventory (M-FPI) in an infertile Chinese sample. J Psychosom Obstet Gynaecol. 2011;32(4):173-81. doi:10.3109/0167482X.2011.609950.

29. Rosenberg M. Society and the Adolescent Self-Image. Sociology. 1965. doi:10. 2307/2575639.

30. Cheng ST, Hamid PN. An error in the use of translated scales: The Rosenberg Self-Esteem Scale for Chinese. Perceptual Motor Skills. 1995;81(2):431-4. doi:10.3758/BF03205466.

31. Urzúa A, Ferrer R, Godoy N, Leppes F, Trujillo C, Osorio C, Caqueo-Urízar A. The mediating effect of self-esteem on the relationship between perceived discrimination and psychological well-being in immigrants. PLoS One. 2018;13(6):e0198413. doi:10.1371/journal.pone.0198413.

32. Hayes A. Introduction to mediation, moderation, and conditional process analysis. J Educ Meas, 2013, 51(3):335-7. doi:10.1111/jedm. 12050.

33. Preacher KJ, Hayes AF. Asymptotic and resampling strategies for assessing and comparing indirect effects in multiple mediator models. Behav Res Methods. 2008;40(3):879-91. doi:10.3758/brm.40.3.879.

34. Tola EN, Eris Yalcin S, Dugan N, Oral B. The association of type D personality and depression with infertility in women. J Psychosom Obstet Gynaecol. 2020;41(2):98-105. doi:10.1080/0167482X.2019.1573224.

35. Biringer E, Howard LM, Kessler U, Stewart R, Mykletun A. Is infertility really associated with higher levels of mental distress in the female population? Results from the North-Trøndelag Health Study and the Medical Birth Registry of Norway. J Psychosom Obstet Gynaecol. 2015;36(2):38-45. doi: 10.3109/0167482X.2014. 992411. 
36. Peterson BD, Sejbaek CS, Pirritano M, Schmidt L. Are severe depressive symptoms associated with infertilityrelated distress in individuals and their partners? Hum Reprod. 2014;29(1):76-82. doi:10.1093/humrep/det412.

37. Ramezanzadeh F, Aghssa MM, Abedinia N, Zayeri F, Khanafshar N, Shariat M, Jafarabadi M. A survey of relationship between anxiety, depression and duration of infertility. BMC Womens Health. 2004;4(1):9. doi:10.1186/1472-6874-4-9.

38. Cousineau TM, Domar AD. Psychological Impacts of Infertility Among Married Women Attending in a Tertiary Hospital, Dhaka. Anwer Khan Modern Medical College Journal. 2018; 9(1):10-4. doi: 10.3329/akmmcj. v9i1.35817.

39. Akyuz A, Sever N. Reasons for infertile couples to discontinue in vitro fertilization (IVF) treatment. Journal of Reproductive Infant Psychology. 2009;27(3):258-68. doi:10.1080/02646830802409652.

40. Verhaak CM, Smeenk JM, Evers AW, Kremer JA, Kraaimaat FW, Braat DD. Women's emotional adjustment to IVF: a systematic review of 25 years of research. Hum Reprod Update. 2007;13(1):27-36. doi:10.1093/humupd/dml040.

41. Cui Y, Li D, Zhou B, Lin Y, Zeng Y. Mediating role of social support between sleep quality, anxiety and depressive symptoms in Chinese women undergoing in vitro fertilization treatment. SAGE Open Med, 2020, 8:2050312120930163. doi:10.11 77/2050312120930163.

42. Segerstrom SC, Miller GE. Psychological stress and the human immune system: a meta-analytic study of 30 years of inquiry. Psychol Bull. 2004;130(4):601-30. doi:10.1037/0033-2909.130.4.601.

43. Li X, Wang K, Huo Y, Zhou M. The effect of infertility-related stress on Chinese infertile females' mental health: The moderating role of marital adjustment. Psych J. 2019;8(2):232-9. doi:10.1002/pchj.255.

44. Gana K, Jakubowska S. Relationship between infertility-related stress and emotional distress and marital satisfaction. J Health Psychol. 2016;21(6):1043-54. doi:10.1177/1359105314544990.

45. Donarelli Z, Lo Coco G, Gullo S, Marino A, Volpes A, Salerno L, Allegra A. Infertility-related stress, anxiety and ovarian stimulation: can couples be reassured about the effects of psychological factors on biological responses to assisted reproductive technology? Reprod Biomed Soc Online, 2016,3: 16-23. doi: j. rbms.2016.10.001.

46. Pedro J, Sobral MP, Mesquita-Guimarães J, Leal C, Costa ME, Martins MV. Couples' discontinuation of fertility treatments: a longitudinal study on demographic, biomedical, and psychosocial risk factors. J Assist Reprod Genet. 2017;34(2):217-24. doi:10.1007/s10815-016-0844-8.

47. Ribeiro RM, Bragiola JVB, Eid LP, Ribeiro RCHM, Sequeira CADC, Pompeo DA. Impact of an intervention through Facebook to strengthen Self-esteem in nursing students. Rev Lat Am Enfermagem. 2020;28:e3237. doi:10.1590/1518-8345. 3215.3237.

\section{Supplementary Files}

This is a list of supplementary files associated with this preprint. Click to download.

- STROBEchecklistcrosssectional.docx 